\title{
On Recurrent Reachability for Continuous Linear Dynamical Systems
}

\author{
Ventsislav Chonev \\ Institute of Science and Technology Austria \\ vchonev@ist.ac.at
}

\author{
Joël Ouaknine James Worrell \\ University of Oxford \\ $\{$ joel,jbw\}@cs.ox.ac.uk
}

\begin{abstract}
The continuous evolution of a wide variety of systems, including continous-time Markov chains and linear hybrid automata, can be described in terms of linear differential equations. In this paper we study the decision problem of whether the solution $\boldsymbol{x}(t)$ of a system of linear differential equations $d \boldsymbol{x} / d t=A \boldsymbol{x}$ reaches a target halfspace infinitely often. This recurrent reachability problem can equivalently be formulated as the following Infinite Zeros Problem: does a real-valued function $f: \mathbb{R}_{\geq 0} \rightarrow \mathbb{R}$ satisfying a given linear differential equation have infinitely many zeros? Our main decidability result is that if the differential equation has order at most 7 , then the Infinite Zeros Problem is decidable. On the other hand, we show that a decision procedure for the Infinite Zeros Problem at order 9 (and above) would entail a major breakthrough in Diophantine Approximation, specifically an algorithm for computing the Lagrange constants of arbitrary real algebraic numbers to arbitrary precision.
\end{abstract}

Categories and Subject Descriptors F.2.m [Analysis of Algorithms and Problem Complexity]: Miscellaneous

Keywords linear dynamical systems, reachability, differential equations, Diophantine Approximation, Skolem problem

\section{Introduction}

A simple type of continuous-time system is one that satisfies a linear differential equation $\frac{d \boldsymbol{x}}{d t}=A \boldsymbol{x}$, where $A$ is an $n \times n$ matrix of rational numbers and $\boldsymbol{x}(t) \in \mathbb{R}^{n}$ gives the system state at time $t$. In particular, such differential equations describe the state evolution of finite-state continuous-time Markov chains (via the so-called rate equation) and the continuous evolution of linear hybrid automata.

A fundamental reachability question in this context is whether $\boldsymbol{x}(t)$ infinitely often reaches a target hyperplane $\boldsymbol{v}^{T} \boldsymbol{x}=0$, where $\boldsymbol{v} \in \mathbb{R}^{n}$ is the normal vector. Such a hyperplane could represent a transition guard in a hybrid automaton or a linear constraint on the state probability distribution of a continuous-time Markov chain (e.g., that the probability to be in a given state is exactly one half).

A solution $\boldsymbol{x}(t)$ to the differential equation $\frac{d \boldsymbol{x}}{d t}=A \boldsymbol{x}$ admits a matrix-exponential representation $\boldsymbol{x}(t)=e^{A t} \boldsymbol{x}(0)$. The problem of whether $\boldsymbol{x}(t)$ reaches the hyperplane $\boldsymbol{v}^{T} \boldsymbol{x}=0$ in- finitely often then reduces to whether $\boldsymbol{v}^{T} \boldsymbol{x}(t)=0$ for infinitely many values of $t \geq 0$. Now the function $f: \mathbb{R}_{>0} \rightarrow \mathbb{R}$ defined by $f(t)=\boldsymbol{v}^{T} \boldsymbol{x}(t)$ can be written as an exponential-polynomial $f(t)=\sum_{j=1}^{k} P_{j}(t) e^{\alpha_{j} t}$, where the $\alpha_{j}$ and the coefficients of each polynomial $P_{j}$ are algebraic numbers. Thus the problem of reaching a hyperplane infinitely often reduces to the Infinite Zeros Problem: given an exponential polynomial $f$, decide whether $f$ has infinitely many non-negative real zeros. Note that since $f$ is an analytic function on the whole real line it only has finitely many zeros in a bounded interval. Thus the Infinite Zeros Problem is equivalent to asking whether the set of zeros of $f$ is unbounded.

This paper is concerned with the decidability of the Infinite Zeros Problem. In order to formulate our main results, recall that exponential polynomials can equivalently be characterised as the solutions of ordinary differential equations

$$
f^{(n)}+a_{n-1} f^{(n-1)}+\ldots+a_{0} f=0
$$

with the coefficients $a_{j}$ and the initial conditions $f^{(j)}(0)$ being real algebraic numbers for $j \in\{0, \ldots, n-1\}$. We say that $f$ has order $n$ if it satisfies a linear differential equation of the form (1).

Our main results concern both decision procedures and hardness results for the Infinite Zeros Problem. We show that the problem is decidable for exponential polynomials of order at most 7 . With regards to hardness, we exhibit a reduction to show that decidability of the Continuous Infinite Zeros Problem for instances of order at least 9 would entail major advancements in the field of Diophantine Approximation, namely the computability of the Lagrange constants of arbitrary real algebraic numbers.

Let us expand on the significance of the above hardness result. Essentially nothing is known about the Lagrange constant of any real algebraic number of degree three or above. For example, it has been a longstanding open problem since the 1930s whether some real algebraic number of degree at least three has strictly positive Lagrange constant and, on the other hand, whether some such number has Lagrange constant 0 (see, e.g., (Guv 2004)). These questions are often formulated in terms of the simple continued fraction expansion of a real number $\alpha$, which has unbounded elements if and only if $\alpha$ has Lagrange constant 0 .

The reader will notice that there is a gap between our decidability and hardness results for exponential polynomials of order 8 . We claim decidability in this case but defer the details to a longer version of this paper.

Another way to calibrate the difficulty of the Infinite Zeros Problem for an exponential polynomial $f(t)=\sum_{j=1}^{k} p_{j}(t) e^{\alpha_{j} t}$ is in terms of the dimension of the $\mathbb{Q}$-vector space spanned by $\left\{\operatorname{Im}\left(\alpha_{j}\right): j=1, \ldots, k\right\}$. We show decidability in case this space is one-dimensional and we observe that the above hardness result already applies in the two-dimensional case. 


\subsection{Related Work}

Closely related to the Infinite Zeros Problem is the problem of whether an exponential polynomial has some zero. This problem is considered in (Bell et al. 2010) under the name Continuous Skolem-Pisot Problem. The techniques considered in the present paper are relevant to the latter problem, but significant extra difficulties arise in this new setting since we cannot discount the behaviour of $f$ on some bounded initial segment of the reals. Our work on the Continuous Skolem-Pisot Problem will be reported elsewhere.

There is a natural discrete analog of the Infinite Zeros Problem: given a linear recurrence sequence, determine whether it has infinitely many zero terms. The decidability of the latter problem was established by Berstel and Mignotte (Berstel and Mignotte 1976). The problem of deciding whether a given linear recurrence sequence has some zero term is called Skolem's Problem. This is a longstanding and celebrated open problem which essentially asks to give an effective proof of the Skolem-Mahler-Lech Theorem for linear recurrences; see, e.g., the exposition of Tao (Tao 2008, Section 3.9).

Macintyre and Wilkie (Macintyre and Wilkie 1996) showed decidability of the first-order theory of $\langle\mathbb{R},+, \times, 0,1,<, \exp \rangle$, the real field with exponentiation, subject to Schanuel's Conjecture in transcendence theory. In this paper we are concerned with the complex exponential function, and we do not use this result. Moreover, although we do make use of transcendence theory, all the results in this paper are unconditional.

\section{Mathematical Background}

\subsection{General Form of a Solution}

We recall some facts about the general form of solutions of ordinary linear differential equations. Consider a homogeneous linear differential equation

$$
f^{(n)}+c_{n-1} f^{(n-1)}+\ldots+c_{0} f=0
$$

of order $n$. The characteristic polynomial of (2) is

$$
\chi(x):=x^{n}+c_{n-1} x^{n-1}+\ldots+c_{0} .
$$

If $\lambda$ is a root of $\chi$ of multiplicity $m$, then the function $f(t)=t^{j} e^{\lambda t}$ satisfies (2) for $j=0,1, \ldots, m-1$. There are $n$ distinct linearly independent solutions of (2) having this form, and these span the space of all solutions.

Let the distinct roots of $\chi$ be $\lambda_{1}, \ldots, \lambda_{k}$, with respective multiplicities $m_{1}, \ldots, m_{k}$. We refer to $\lambda_{1}, \ldots, \lambda_{k}$ as the characteristic roots of the differential equation. We also refer to the characteristic roots of maximum real part as dominant. Write $\lambda_{j}=r_{j}+i a_{j}$ for real algebraic numbers $r_{j}, a_{j}, j=1, \ldots, k$. It follows from the discussion above that, given real algebraic initial values of $f(0), f^{\prime}(0), \ldots, f^{(n-1)}(0)$, the uniquely defined solution $f$ of (2) can be written in one of the following three equivalent forms.

1. As an exponential polynomial

$$
f(t)=\sum_{j=1}^{k} P_{j}(t) e^{\lambda_{j} t}
$$

where each $P_{j}$ is a polynomial with (complex) algebraic coefficients and degree at most $m_{j}-1$.

2. As a function of the form

$$
f(t)=\sum_{j=1}^{k} e^{r_{j} t}\left(P_{j}(t) \cos \left(a_{j} t\right)+Q_{j}(t) \sin \left(a_{j} t\right)\right)
$$

where the polynomials $P_{j}, Q_{j}$ have real algebraic coefficients and degrees at most $m_{j}-1$.
3. As a function of the form

$$
f(t)=\sum_{j=1}^{k} e^{r_{j} t} \sum_{l=0}^{m_{l}-1} b_{j, l} t^{l} \cos \left(a_{j} t+\varphi_{j, l}\right)
$$

where $b_{j, l}$ is real algebraic and $e^{i \varphi_{j, l}}$ algebraic for each $j, l$.

We refer the reader to (Bell et al. 2010, Theorem 7) for details.

\subsection{Number-theoretic tools}

Throughout this paper we denote by $\mathbb{A}$ the set of algebraic numbers. Recall that a standard way to represent an algebraic number $\alpha$ is by its minimal polynomial $M$ and a numerical approximation of sufficient accuracy to distinguish $\alpha$ from the other roots of $M$ (Cohen 1993, Section 4.2.1). Given two algebraic numbers $\alpha$ and $\beta$ under this representation, the Field Membership Problem is to determine whether $\beta \in \mathbb{Q}(\alpha)$ and, if so, to return a polynomial $P$ with rational coefficients such that $\beta=P(\alpha)$. This problem can be decided using the LLL algorithm, see (Cohen 1993, Section 4.5.4).

Given the characteristic polynomial $\chi$ of a linear differential equation we can compute approximations to each of its roots $\lambda_{1}, \ldots, \lambda_{n}$ to within an arbitrarily small additive error (Pan 1996). Moreover, by repeatedly using an algorithm for the Field Membership Problem we can compute a primitive element $\theta$ for the splitting field of $\chi$ and representations of $\lambda_{1}, \ldots, \lambda_{n}$ as polynomials in $\theta$. Thereby we can determine maximal $\mathbb{Q}$-linearly independent subsets of $\left\{\operatorname{Re}\left(\lambda_{j}\right): 1 \leq j \leq n\right\}$ and $\left\{\operatorname{Im}\left(\lambda_{j}\right): 1 \leq j \leq n\right\}$.

We now move to some techniques from Transcendental Number Theory on which our results depend in a critical way. The following theorem was originally proven in 1934 by A. Gelfond (Gelfond 1934; Gelfond and Vinogradov 1934) and independently by T. Schneider (Schneider 1935a.b), settling Hilbert's seventh problem in the affirmative.

Theorem 1. (Gelfond-Schneider) If a and b are algebraic numbers with $a \neq 0,1$ and $b \notin \mathbb{Q}$, then $a^{b}$ is transcendental.

The following lemma, proven in (Bell et al. 2010), is a useful consequence of the powerful Baker's Theorem (Baker 1975, Theorem 3.1):

Lemma 2. Bell et al. 2010, Lemma 13) Let $a, b \in \mathbb{R} \cap \mathbb{A}$ be linearly independent over $\mathbb{Q}$ and let $\varphi_{1}, \varphi_{2}$ be logarithms of algebraic numbers, that is, $e^{i \varphi_{1}}, e^{i \varphi_{2}} \in \mathbb{A}$. There exist effective constants $C, N, T>0$ such that for all $t \geq T$, at least one of $1-\cos \left(a t+\varphi_{1}\right)>C / t^{N}$ and $1-\cos \left(b t+\varphi_{2}\right)>C / t^{N}$ holds.

Another necessary tool is a version of Kronecker's well-known Theorem in Diophantine Approximation.

Theorem 3. (Kronecker, appears in (Hardy and Wright 1999)) Let $\lambda_{1}, \ldots, \lambda_{m}$ and $x_{1}, \ldots, x_{m}$ be real numbers. Suppose that for all integers $u_{1}, \ldots, u_{m}$ such that $u_{1} \lambda_{1}+\cdots+u_{m} \lambda_{m} \in \mathbb{Z}$, we also have $u_{1} x_{1}+\cdots+u_{m} x_{m} \in \mathbb{Z}$, that is, all integer relations among the $\lambda_{j}$ also hold among the $x_{j}$ (modulo $\mathbb{Z}$ ). Then for all $\epsilon>0$, there exist $p \in \mathbb{Z}^{m}$ and $n \in \mathbb{N}$ such that $\left|n \lambda_{j}-x_{j}-p_{j}\right|<\epsilon$ for all $1 \leq j \leq m$. In particular, if $1, \lambda_{1}, \ldots, \lambda_{m}$ are linearly independent over $\mathbb{Z}$, then there exist such $n \in \mathbb{N}$ and $p \in \mathbb{Z}^{m}$ for all $x \in \mathbb{R}^{m}$ and $\epsilon>0$.

A direct consequence is the following:

Lemma 4. Let $a_{1}, \ldots, a_{m} \in \mathbb{R} \cap \mathbb{A}$ be linearly independent over $\mathbb{Q}$ and let $\varphi_{1}, \ldots, \varphi_{m} \in \mathbb{R}$. Write $x \bmod 2 \pi$ to denote $\min _{k \in \mathbb{Z}}|x+2 k \pi|$ for any $x \in \mathbb{R}$. Then the image of the mapping $h(t): \mathbb{R}_{\geq 0} \rightarrow[0,2 \pi)^{m}$ given by

$$
h(t)=\left(\left(a_{1} t+\varphi_{1}\right) \bmod 2 \pi, \ldots,\left(a_{m} t+\varphi_{m}\right) \bmod 2 \pi\right)
$$


is dense in $[0,2 \pi)^{m}$. Moreover, the set

$$
\left\{h(t) \mid\left(a_{1} t+\varphi_{1}\right) \bmod 2 \pi=0\right\}
$$

is dense in $\{0\} \times[0,2 \pi)^{m-1}$.

Proof. For the first part of the claim, note that the linear independence of $1, a_{1} / 2 \pi, \ldots, a_{m} / 2 \pi$ follows from the linear independence of $a_{1}, \ldots, a_{m}$ and the transcendence of $\pi$. Then by Kronecker's Theorem, the restriction $\{h(t) \mid t \in \mathbb{N}\}$ is dense in $[0,2 \pi)^{m}$, so certainly the whole image of $h(t)$ must also be dense in $[0,2 \pi)^{m}$. For the second part, the trajectory $h(t)$ has zero first coordinate precisely when $t=-\varphi_{1} / a_{1}+2 n \pi$ for some $n \in \mathbb{Z}$, at which times the trajectory is

$$
\begin{aligned}
g(n) & \stackrel{\text { def }}{=} h\left(\frac{-\varphi_{1}}{a_{1}}+2 n \pi\right) \\
& =\{0\} \times\left(n \frac{2 \pi a_{j}}{a_{1}}+\frac{a_{1} \varphi_{j}-\varphi_{1} a_{j}}{a_{1}} \bmod 2 \pi\right)_{2 \leq j \leq m}
\end{aligned}
$$

As before, we have that $\left\{1,2 \pi a_{2} / a_{1}, \ldots, 2 \pi a_{m} / a_{1}\right\}$ are linearly independent over $\mathbb{Q}$ from the linear independence of $a_{1}, \ldots, a_{m}$ and the transcendence of $\pi$, so applying Kronecker's Theorem to the last $m-1$ components of this discrete trajectory yields the second part of the claim.

\subsection{First-Order Theory of the Reals}

We denote by $\mathcal{L}$ the first-order language $\langle\mathbb{R},+, \times, 0,1,<\rangle$. Atomic formulas in this language are of the form $P\left(x_{1}, \ldots, x_{n}\right)=0$ and $P\left(x_{1}, \ldots, x_{n}\right)>0$ for $P \in \mathbb{Z}\left[x_{1}, \ldots, x_{n}\right]$ a polynomial with integer coefficients. A set $X \subseteq \mathbb{R}^{n}$ is definable in $\mathcal{L}$ if there exists some $\mathcal{L}$-formula $\phi(\bar{x})$ with free variables $\bar{x}$ which holds precisely for valuations in $X$. Analogously, a function is definable if its graph is a definable set.

We denote by $T h(\mathbb{R})$ the first-order theory of the reals, that is, the set of all valid sentences in the language $\mathcal{L}$. It is worth remarking that any real algebraic number is readily definable within $\mathcal{L}$ using its minimal polynomial and a rational approximation to distinguish it from the other roots. Thus, we can treat real algebraic numbers constants as built into the language and use them freely in the construction of formulas. A celebrated result due to Tarski (Tarski 1951) is that the first-order theory of the reals admits quantifier elimination: that each formula $\phi_{1}(\bar{x})$ in $\mathcal{L}$ is equivalent to some effectively computable formula $\phi_{2}(\bar{x})$ which uses no quantifiers. This immediately entails the decidability of $T h(\mathbb{R})$. It also follows that sets definable in $\mathcal{L}$ are precisely the semialgebraic sets. Tarski's original result had non-elementary complexity, but improvements followed, culminating in the detailed analysis of Renegar (Renegar 1992).

Decidability and geometrical properties of definable sets in the first-order theory of the structure $\mathcal{L}_{\exp }=\langle\mathbb{R},+, \times, 0,1,<, \exp \rangle$, the reals with exponentiation, have been explored by a number of authors. Most notably, Wilkie (Wilkie 1996) showed that the theory is o-minimal and Macintyre and Wilkie (Macintyre and Wilkie 1996) showed that if Schanuel's conjecture is true then the theory is decidable. We will not need the above two results in this paper, however we use the following, which is very straightforward to establish directly.

Proposition 5. There is a procedure that, given a semi-algebraic set $S \subseteq \mathbb{R}^{k}$ and real algebraic numbers $a_{1}, \ldots, a_{k}$, returns an integer $T$ such that $\left\{t \geq 0:\left(e^{a_{1} t}, \ldots, e^{a_{k} t}\right) \in S\right\}$ either contains the interval $(T, \infty)$ or is disjoint from $(T, \infty)$. The procedure also decides which of these two eventualities is the case.

Proof. Consider a polynomial $P \in \mathbb{Z}\left[u_{1}, \ldots, u_{k}\right]$. For suitably large $t$ the sign of $P\left(e^{a_{1} t}, \ldots, e^{a_{k} t}\right)$ is identical to the sign of the coefficient of the dominant term in the expansion of $P\left(e^{a_{1} t}, \ldots, e^{a_{k} t}\right)$ as an exponential polynomial. It follows that the sign of $P\left(e^{a_{1} t}, \ldots, e^{a_{k} t}\right)$ is eventually constant. It is moreover clear that one can effectively compute a threshold beyond which the sign of $P\left(e^{a_{1} t}, \ldots, e^{a_{k} t}\right)$ remains the same. Since the set $S$ is defined by a Boolean combination of inequalities $P\left(u_{1}, \ldots, u_{k}\right) \sim 0$, for $\sim \in\{<,=\}$, the proposition immediately follows.

\subsection{Useful Results About Exponential Polynomials}

We restate two useful theorems due to Bell et al. (Bell et al. 2010).

Theorem 6. (Bell et al.2010, Theorem 12) Exponential polynomials with no real dominant characteristic roots have infinitely many zeros.

Theorem 7. Bell et al. 2010, Theorem 15) Suppose we are given an exponential polynomial whose dominant characteristic roots are simple, at least four in number and have imaginary parts linearly independent over $\mathbb{Q}$. Then the existence of infinitely many zeros is decidable.

\section{One Linearly Independent Oscillation}

In this section we consider exponential polynomials $f(t)=$ $\sum_{j=1}^{k} P_{j}(t) e^{\lambda_{j} t}$ under the assumption that the span of $\left\{\operatorname{Im}\left(\lambda_{j}\right)\right.$ : $j=1, \ldots, k\}$ is a one-dimensional $\mathbb{Q}$-vector space. In this case we can use fundamental geometric properties of semi-algebraic sets to decide whether or not $f$ has finitely many zeros.

Theorem 8. Let $f(t)=\sum_{j=1}^{k} P_{j}(t) e^{\lambda_{j} t}$ be an exponential polynomial such that the span of $\left\{\operatorname{Im}\left(\lambda_{j}\right): j=1, \ldots, k\right\}$ is a onedimensional $\mathbb{Q}$-vector space. Then the existence of infinitely many zeros of $f$ is decidable.

Proof. Write $\lambda_{j}=a_{j}+i b_{j}$, where $a_{j}, b_{j}$ are real algebraic numbers for $j=1, \ldots, k$. By assumption there is a single real algebraic number $b$ such that each $b_{j}$ is an integer multiple of $b$. Recall that for each integer $n$, both $\cos (n b t)$ and $\sin (n b t)$ can be written as polynomials in $\sin (b t)$ and $\cos (b t)$ with integer coefficients. Using this fact we can write $f$ in the form

$$
f(t)=Q\left(t, e^{a_{1} t}, \ldots, e^{a_{k} t}, \cos (b t), \sin (b t)\right),
$$

for some multivariate polynomial $Q$ with algebraic coefficients.

Now consider the semi-algebraic set

$$
E:=\left\{(\boldsymbol{u}, s) \in \mathbb{R}^{k+2}: Q\left(u_{0}, \ldots, u_{k}, \frac{1-s^{2}}{1+s^{2}}, \frac{2 s}{1+s^{2}}\right)=0\right\} .
$$

Recall that $\left\{\left(\frac{1-s^{2}}{1+s^{2}}, \frac{2 s}{1+s^{2}}\right): s \in \mathbb{R}\right\}$ comprises all points in the unit circle in $\mathbb{R}^{2}$ except $(-1,0)$. Indeed, given $\theta \in(-\pi, \pi)$, setting $s:=\tan (\theta / 2)$ we have $\cos (\theta)=\frac{1-s^{2}}{1+s^{2}}$ and $\sin (\theta)=$ $\frac{2 s}{1+s^{2}}$. It follows that $f(t)=0$ and $\cos (b t) \stackrel{1}{\neq}-1$ imply that $\left(t, e^{a_{1} t}, \ldots, e^{a_{k} t}, \tan (b t / 2)\right) \in E$.

By the Cell Decomposition Theorem for semi-algebraic sets (Marker 2002), there are semi-algebraic sets $C_{1}, \ldots, C_{m} \subseteq \mathbb{R}^{k+2}$, $D_{1}, \ldots, D_{m} \subseteq \mathbb{R}^{k+1}$, and continuous semi-algebraic functions $\xi_{j}, \xi_{j}^{(1)}, \xi_{j}^{(2)}: D_{j} \rightarrow \mathbb{R}$ such that $E$ can be written as a disjoint union $E=C_{1} \cup \ldots \cup C_{m}$, where either

$$
C_{j}=\left\{(\boldsymbol{u}, s) \in \mathbb{R}^{k+2}: \boldsymbol{u} \in D_{j} \wedge s=\xi_{j}(\boldsymbol{u})\right\}
$$

or

$C_{j}=\left\{(\boldsymbol{u}, s) \in \mathbb{R}^{k+2}: \boldsymbol{u} \in D_{j} \wedge \xi_{j}^{(1)}(\boldsymbol{u})<s<\xi_{j}^{(2)}(\boldsymbol{u})\right\}$ 


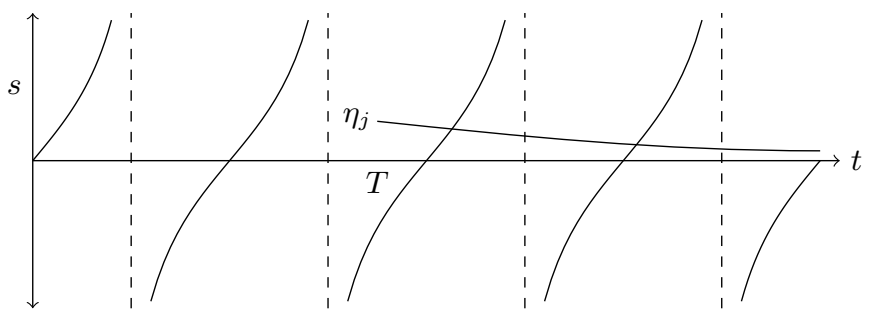

Figure 1. Intersection points of $\eta_{j}(t)$ and $\tan (b t / 2)$.

Moreover such a decomposition is computable from $E$. Clearly then

$\{t \in \mathbb{R}: f(t)=0\} \subseteq \bigcup_{j=1}^{m}\left\{t \in \mathbb{R}:\left(t, e^{a_{1} t}, \ldots, e^{a_{k} t}\right) \in D_{j}\right\} \cup Z$,

where $Z:=\{t \in \mathbb{R}: \cos (b t)=-1\}$.

The restriction of the exponential polynomial $f$ to $Z$ is given by $f(t)=Q\left(t, e^{a_{1} t}, \ldots, e^{a_{k} t},-1,0\right)$. Since this expression is a linear combination of terms of the form $t^{j} e^{r t}$ for real algebraic $r$, for sufficiently large $t$ the sign of $f(t)$ is determined by the sign of the coefficient of the dominant term. Thus $f$ is either identically zero on $Z$ (in which case $f$ has infinitely many zeros) or there exists some threshold $T$ such that all zeros of $f$ in $Z$ lie in the interval $[0, T]$.

We now consider zeros of $f$ that do not lie in $Z$. There are two cases. First suppose that each set $\left\{t \in \mathbb{R}:\left(t, e^{a_{1} t}, \ldots, e^{a_{k} t}\right) \in\right.$ $\left.D_{j}\right\}$ is bounded for $j=1, \ldots, m$. In this situation, using Proposition 5] we can obtain an upper bound $T$ such that if $f(t)=$ 0 then $t<T$. On the other hand, if some set $\left\{t \in \mathbb{R}_{\geq 0}\right.$ : $\left.\left(t, e^{a_{1} t}, \ldots, e^{a_{k} t}\right) \in D_{j}\right\}$ is unbounded then, by Proposition 5 . it contains an infinite interval $(T, \infty)$. We claim that in this case $f$ must have infinitely many zeros $t \geq 0$. We first give the argument in the case $C_{j}$ satisfies (3).

Define $\eta_{j}(t)=\xi_{j}\left(t, e^{a_{1} t}, \ldots, e^{a_{k} t}\right)$ for $t \in(T, \infty)$. Then for $t \in(T, \infty) \backslash Z$,

$$
\begin{aligned}
f(t)=0 & \Longleftrightarrow\left(t, e^{a_{1} t}, \ldots, e^{a_{k} t}, \tan (b t / 2)\right) \in C_{j} \\
& \Longleftrightarrow\left(t, e^{a_{1} t}, \ldots, e^{a_{k} t}\right) \in D_{j} \wedge \eta_{j}(t)=\tan (b t / 2) .
\end{aligned}
$$

In other words, $f$ has a zero at each point $t \in(T, \infty) \backslash Z$ at which the graph of $\eta_{j}$ intersects the graph of $\tan (b t / 2)$. Since $\eta_{j}$ is continuous there are clearly infinitely many such intersection points, see Figure 1

The case when $C_{j}$ satisifes (4) is handled similarly. In fact, this case cannot arise at all, since by the above argument, if $C_{j}$ satisfies (4), then $f$ has a non-trivial interval of zeros. This is impossible, since $f$ is analytic, and hence has only isolated zeros. This completes the proof.

\section{Decidability Results up to Order 7}

We now shift our attention to instances of the Infinite Zeros Problem of low order. In particular, for exponential polynomials corresponding to differential equations of order at most 7 , we establish decidability of the Infinite Zeros Problem.

Theorem 9. The Infinite Zeros Problem is decidable for differential equations of order at most 7 .

Proof. Suppose we are given an exponential polynomial $f$ of order at most 7 . Sort the characteristic roots according to their real parts, and let $r_{j}$ denote throughout the $j$-th largest real part of a characteristic root. We will refer to the characteristic roots of maximum real part as the dominant characteristic roots. Let also $m u l(\lambda)$ denote the multiplicity of $\lambda$ as a root of the characteristic polynomial of $f$.

We will now perform a case analysis on the number of dominant characteristic roots. By Theorem 6, it is sufficient to confine our attention to exponential polynomials with an odd number of dominant characteristic roots. Throughout, we rely on known general forms of solutions to ordinary linear differential equations, outlined in Section 2.1

Case I. Suppose first that there is only one dominant, necessarily real, root $r$. Then if we divide $f$ by $e^{r t}$, we have:

$$
\frac{f(t)}{e^{r t}}=P_{1}(t)+\mathcal{O}\left(e^{\left(r_{2}-r\right) t}\right)
$$

as the contribution of the non-dominant roots shrinks exponentially, relative to that of the dominant root. Thus, for large $t \geq 0$, the sign of $f(t)$ matches the sign of the leading coefficient of $P_{1}(t)$, so $f$ cannot have infinitely many zeros.

Case II. We now move to the case of three dominant characteristic roots: $r$ and $r \pm i a$, so that

$\frac{f(t)}{e^{r t}}=P_{1}(t)+P_{2}(t) \cos (a t)+P_{3}(t) \sin (a t)+\mathcal{O}\left(e^{\left(r_{2}-r\right) t}\right)$,

where $P_{1}, P_{2}, P_{3} \in(\mathbb{R} \cap \mathbb{A})[x]$ have degrees $d_{1} \stackrel{\text { def }}{=} \operatorname{deg}\left(P_{1}\right) \leq$ $\operatorname{mul}(r)-1$ and $d_{2} \stackrel{\text { def }}{=} \operatorname{deg}\left(P_{2}\right)=\operatorname{deg}\left(P_{3}\right) \leq \operatorname{mul}(r \pm a i)$.

Case IIa. Suppose $d_{1}>d_{2}$. Now, it is easy to see that for large $t$ the sign of $f(t)$ matches the sign of the leading coefficient $p_{1}$ of $P_{1}$ :

$$
\frac{f(t)}{e^{r t} t^{d_{1}}}=p_{1}+\mathcal{O}(1 / t)+\mathcal{O}\left(e^{\left(r_{2}-r\right) t}\right),
$$

so clearly some bound $T$ exists such that $t>T \Rightarrow f(t) \neq 0$. Similarly, if $d_{2}>d_{1}$, then $f(t)$ clearly has infinitely many zeros. Indeed, if $p_{2}, p_{3}$ are the leading coefficients of $P_{2}, P_{3}$, respectively, then we have:

$$
\begin{aligned}
\frac{f(t)}{e^{r t} t^{d_{2}}} & =p_{2} \cos (a t)+p_{3} \sin (a t)+\mathcal{O}(1 / t)+\mathcal{O}\left(e^{\left(r_{2}-r\right) t}\right) \\
& =\frac{\cos (a t+\varphi)}{\sqrt{p_{2}^{2}+p_{3}^{2}}}+\mathcal{O}(1 / t)+\mathcal{O}\left(e^{\left(r_{2}-r\right) t}\right)
\end{aligned}
$$

where $\varphi \in[0,2 \pi)$ with $\tan (\varphi)=-p_{3} / p_{2}$, so $f$ is infinitely often positive and infinitely often negative.

Thus, we can now assume $d_{1}=d_{2}$. Notice that since the order of our exponential polynomial is no greater than 7 , we must have $d_{1}=d_{2} \leq 2$.

Case II $b$. Suppose that $d_{1}=d_{2}=2$. Then our function is of the form

$\frac{f(t)}{e^{r t}}=t\left(A \cos \left(a t+\varphi_{1}\right)+B\right)+\left(C \cos \left(a t+\varphi_{2}\right)+D\right)+e^{\left(r_{2}-r\right) t} F$,

for constants $A, B, C, D, F, a \in \mathbb{R} \cap \mathbb{A}$ with $a>0$ and $e^{i \varphi_{1}}, e^{i \varphi_{2}} \in$ $\mathbb{A}$. In this case, Theorem 9 follows from Lemma 16 in Section 4.4

Case IIc. Suppose that $d_{1}=d_{2}=1$, so that

$$
\frac{f(t)}{e^{r t}}=A_{1} \cos \left(a t+\varphi_{1}\right)+A_{2}+e^{\left(r_{2}-r\right) t} F_{1}(t),
$$

where $A_{1}, A_{2}, a \in \mathbb{R} \cap \mathbb{A}, a>0, e^{i \varphi_{1}} \in \mathbb{A}$ and $F_{1}$ is an exponential polynomial with dominant characteristic root whose real part is 0 . Consider first the magnitudes of $A_{1}$ and $A_{2}$. If $\left|A_{1}\right|>\left|A_{2}\right|$, then the term $A_{1} \cos \left(a t+\varphi_{1}\right)$ makes $f$ change sign infinitely often, so $f$ must have infinitely many zeros. On the other hand, if $\left|A_{1}\right|<\left|A_{2}\right|$, then $f$ is clearly ultimately positive or ultimately negative, depending on the sign of $A_{2}$. The remaining case is that $\left|A_{1}\right|=\left|A_{2}\right|$. Dividing $f$ by $A_{2}$, replacing $\varphi_{1}$ by $\varphi_{1}+\pi$ if needed and scaling constants by $A_{2}$ as necessary, we can assume 
the function has the form:

$$
\frac{f(t)}{e^{r t}}=1-\cos \left(a t+\varphi_{1}\right)+e^{\left(r_{2}-r\right) t} F_{1}(t) .
$$

We now enumerate the possibilities for the dominant characteristic roots of the exponential polynomial $F_{1}$, that is, the characteristic roots of $f$ with second-largest real part. Since $f$ has order at most 7 , there are the following cases to consider:

- $F_{1}$ has four simple, necessarily complex, dominant roots, so that

$$
\begin{aligned}
\frac{f(t)}{e^{r t}}=1 & -\cos \left(a t+\varphi_{1}\right) \\
& +e^{\left(r_{2}-r\right) t}\left(B \cos \left(b t+\varphi_{2}\right)+C \cos \left(c t+\varphi_{3}\right)\right),
\end{aligned}
$$

where $B, C, b, c \in \mathbb{R} \cap \mathbb{A}$ with $b, c>0$ and $e^{i \varphi_{2}}, e^{i \varphi_{3}} \in \mathbb{A}$. In this case, Theorem 9 follows from Lemma 12 in Section 4.1

- $F_{1}$ has some subset of one real and two complex numbers as dominant roots, all simple, so that

$$
\begin{aligned}
\frac{f(t)}{e^{r t}}=1 & -\cos \left(a t+\varphi_{1}\right) \\
& +e^{\left(r_{2}-r\right) t}\left(B \cos \left(b t+\varphi_{2}\right)+C\right)+e^{\left(r_{3}-r\right) t} F_{2}(t),
\end{aligned}
$$

where $B, C, b \in \mathbb{R} \cap \mathbb{A}, b>0, e^{i \varphi_{2}} \in \mathbb{A}$ and $F_{2}$ is an exponential polynomial with dominant characteristic root whose real part is 0 . In this case, Theorem 9 follows from Lemma 11 in Section 4.1

- $F_{1}$ has a repeated real and possibly two simple complex dominant roots, so that

$$
\begin{aligned}
\frac{f(t)}{e^{r t}}=1 & -\cos \left(a t+\varphi_{1}\right) \\
& +e^{\left(r_{2}-r\right) t}\left(B \cos \left(b t+\varphi_{2}\right)+P(t)\right)+e^{\left(r_{3}-r\right) t} F_{2}(t),
\end{aligned}
$$

where $B, b \in \mathbb{R} \cap \mathbb{A}, b>0, e^{i \varphi_{2}} \in \mathbb{A}$, and $P \in(\mathbb{R} \cap \mathbb{A})[x]$ is non-constant. Now, if the leading coefficient of $P$ is negative, then $f$ will be infinitely often negative (consider large times $t$ such that $\left.\cos \left(a t+\varphi_{1}\right)=1\right)$ and infinitely often positive (consider large times $t$ such that $\cos \left(a t+\varphi_{1}\right)=0$ ), so $f$ must have infinitely many zeros. On the other hand, if the leading coefficient of $P$ is positive, then it is easy to see that $f$ is ultimately positive.

- $F_{1}$ has a repeated pair of complex roots, so that

$$
\begin{aligned}
\frac{f(t)}{e^{r t}}=1 & -\cos \left(a t+\varphi_{1}\right) \\
& +e^{\left(r_{2}-r\right) t}\left(B t \cos \left(b t+\varphi_{2}\right)+C \cos \left(b t+\varphi_{3}\right)\right),
\end{aligned}
$$

where $B, C, b \in \mathbb{R} \cap \mathbb{A}, b>0$ and $e^{i \varphi_{2}}, e^{i \varphi_{3}} \in \mathbb{A}$. In this case, Theorem 9 follows from Lemma 13 in Section 4.1

Case III. We now consider the case of five dominant characteristic roots. Let these be $r, r \pm a i$ and $r \pm b i$. If $r \pm a i$ are repeated, i.e., $\operatorname{mul}(r \pm a i) \geq 2$, then we must have $\operatorname{mul}(r)=$ $\operatorname{mul}(r \pm b i)=1$, since otherwise the order of our exponential polynomial exceeds 7 . Then by an argument analogous to Case IIa above, $f$ must have infinitely many zeros. The situation is symmetric when $\operatorname{mul}(r \pm b i) \geq 2$. Similarly, if $\operatorname{mul}(r) \geq 2$, then $\operatorname{mul}(r \pm a i)=\operatorname{mul}(r \pm b i)=1$, since otherwise the instance exceeds order 7 . Then by the same argument as in Case II $a, f$ is ultimately positive or ultimately negative. Thus, we may assume that all the dominant roots are simple, so the exponential polynomial is of the form:

$$
\frac{f(t)}{e^{r t}}=A \cos \left(a t+\varphi_{1}\right)+B \cos \left(b t+\varphi_{2}\right)+C+e^{\left(r_{2}-r\right) t} F(t),
$$

where $A, B, C, a, b \in \mathbb{R} \cap \mathbb{A}, a, b>0, e^{i \varphi_{1}}, e^{i \varphi_{2}} \in \mathbb{A}$ and $F$ is an exponential polynomial of order at most 2 whose dominant characteristic roots have real part equal to 0 . In this case, Theorem 9 follows from Lemma 14 in Section 4.2

Case IV. Finally, suppose there are seven dominant characteristic roots: $r, r \pm a i, r \pm b i$ and $r \pm c i$. Since we are limiting ourselves to instances of order 7 , these roots must all be simple, and there can be no other characteristic roots. Thus, the exponential polynomial has the form

$\frac{f(t)}{e^{r t}}=A \cos \left(a t+\varphi_{1}\right)+B \cos \left(b t+\varphi_{2}\right)+C \cos \left(c t+\varphi_{3}\right)+D$, with $A, B, C, D, a, b, c \in \mathbb{R} \cap \mathbb{A}, a, b, c>0$ and $e^{i \varphi_{1}}, \ldots, e^{i \varphi_{3}} \in$ $\mathbb{A}$. In this case, Theorem 9 follows from Lemma 15 in Section 4.3

In the remainder of this section, we provide the technical lemmas invoked throughout the proof of Theorem 9

\subsection{One dominant oscillation}

Lemma 10. Let $A, B, a, b, r \in \mathbb{R} \cap \mathbb{A}$ where $a, b, r>0$. Let $\varphi_{1}, \varphi_{2} \in \mathbb{R}$ be such that $e^{i \varphi_{1}}, e^{i \varphi_{2}} \in \mathbb{A}$. Suppose also that $a, b$ are linearly dependent over $\mathbb{Q}$ and that whenever $1-\cos \left(a t+\varphi_{1}\right)=0$, it holds that $A \cos \left(b t+\varphi_{2}\right)+B>0$. Define the function

$$
f(t)=1-\cos \left(a t+\varphi_{1}\right)+e^{-r t}\left(A \cos \left(b t+\varphi_{2}\right)+B\right) .
$$

Then $f(t)=\Omega\left(e^{-r t}\right)$, that is, there exist effective constants $T \geq 0$ and $c>0$ such that for $t \geq T$, we have $f(t) \geq c e^{-r t}$.

Proof. The case of $A=0$ is easy: by the premise of the Lemma, we have $B>0$ and then $f(t) \geq B e^{-r t}$ for all $t$. Thus, assume $A \neq 0$ throughout. Let the linear dependence between $a, b$ be given by $a n_{1}-b n_{2}=0$ for $n_{1}, n_{2} \in \mathbb{N}$ coprime and let $\mathcal{C}$ be the equivalence class of $-\varphi_{1} / a$ modulo $2 \pi / a$, that is,

$$
\mathcal{C} \stackrel{\text { def }}{=}\left\{\frac{-\varphi_{1}+2 k \pi}{a} \mid k \in \mathbb{Z}\right\} .
$$

We will refer to $\mathcal{C}$ as the set of critical points throughout.

It is clear that at critical points, we have $1-\cos \left(a t+\varphi_{1}\right)=0$. Moreover, the linear dependence of $a, b$ entails that for each fixed value of $(\cos (a t), \sin (a t))$, there are only finitely many possible values for $(\cos (b t), \sin (b t))$. Indeed, we have

$$
e^{i b t} \in\left\{\omega e^{i a t n_{1}} \mid \omega \text { an } n_{2} \text {-th root of unity }\right\},
$$

so in particular, for $t \in \mathcal{C}$, we have

$$
e^{i b t} \in\left\{\omega e^{-i n_{1} \varphi_{1}} \mid \omega \text { an } n_{2} \text {-th root of unity }\right\} .
$$

Thus, the possible values of $(\cos (b t), \sin (b t))$ for $t$ critical are algebraic and effectively computable. Let $M \stackrel{\text { def }}{=} \min \{A \cos (b t+$ $\left.\left.\varphi_{2}\right)+B \mid t \in \mathcal{C}\right\}$. By the premise of the Lemma, we have $M>0$.

Let $t_{1}, t_{2}, \ldots, t_{j}, \ldots$ be the non-negative critical points. Note that by construction we have $\left|t_{j}-t_{j-1}\right|=2 \pi / a$. For each $t_{j}$, define the critical region to be the interval $\left[t_{j}-\delta, t_{j}+\delta\right]$, where

$$
\delta \stackrel{\text { def }}{=} \frac{M}{2|A| b} .
$$

Let $g(t) \stackrel{\text { def }}{=} A \cos \left(b t+\varphi_{2}\right)+B$ and notice that $g^{\prime}(t) \leq|A| b$ everywhere. We first prove the claim for $t$ inside critical regions: suppose $t$ lies in a critical region and let $j$ minimise $\left|t-t_{j}\right| \leq \delta$. Then by the Mean Value Theorem, we have

so

$$
\left|g(t)-g\left(t_{j}\right)\right| \leq\left|t-t_{j}\right||A| b \leq \delta|A| b=\frac{M}{2},
$$

$$
g(t) \geq g\left(t_{j}\right)-\frac{M}{2} \geq \frac{M}{2},
$$


whence $f(t) \geq e^{-r t} g(t) \geq M e^{-r t} / 2=\Omega\left(e^{-r t}\right)$.

Now suppose $t$ is outside all critical regions and let $j$ minimise $\left|t-t_{j}\right|$. Since the distance between critical points is $2 \pi / a$ by construction, we have $a\left|t-t_{j}\right| \leq \pi$. Therefore,

$$
\begin{aligned}
1-\cos \left(a t+\varphi_{1}\right) & =1-\cos \left(a t-a t_{j}\right) \geq \frac{\left|a\left(t-t_{j}\right)\right|^{2}}{2} \\
& >\frac{(a \delta)^{2}}{2}=\frac{a^{2} M^{2}}{8|A|^{2} b^{2}}>0 .
\end{aligned}
$$

Thus, there exists a computable constant $D>0$ such that $f(t)=$ $1-\cos \left(a t+\varphi_{1}\right)+e^{-r t} g(t) \geq D$ for all large enough $t$ outside critical regions.

Combining the two results, we have $f(t)=\Omega\left(e^{-r t}\right)$ everywhere.

Lemma 11. Let $C, D, a, b, r_{1}, r_{2}$ be real algebraic numbers such that $a, b, r_{1}, r_{2}>0$ and $C, D$ are not both 0 . Let also $\varphi_{1}, \varphi_{2} \in \mathbb{R}$ be such that $e^{i \varphi_{1}}, e^{i \varphi_{2}} \in \mathbb{A}$. Define the exponential polynomial $f$ by

$$
\begin{aligned}
f(t)= & -\cos \left(a t+\varphi_{1}\right) \\
& +e^{-r_{1} t}\left(C \cos \left(b t+\varphi_{2}\right)+D\right)+e^{-\left(r_{1}+r_{2}\right) t} F(t) .
\end{aligned}
$$

Here $F$ is an exponential polynomial whose dominant characteristic roots are purely imaginary. Suppose also that $f$ has order at most 7 . Then it is decidable whether $f$ has infinitely many zeros.

Proof. Notice that the dominant term of $f$ is always non-negative, so the function is positive for arbitrarily large $t$. Thus, $f(t)=0$ for some $t$ if and only if $f(t) \leq 0$ for some $t$, and analogously, $f$ has infinitely many zeros if and only if $f(t) \leq 0$ infinitely often. We can eliminate the case $|D|>|C|$, since then $f$ is clearly ultimately positive or oscillating, depending on the sign of $D$. Thus, we can assume $|D| \leq|C|$.

We now consider two cases, depending on whether $a / b \in \mathbb{Q}$.

Case I. Suppose first that $a, b$ are linearly independent over $\mathbb{Q}$. By Lemma 4 the trajectory $\left(a t+\varphi_{1} \bmod 2 \pi, b t+\varphi_{2} \bmod 2 \pi\right)$ is dense in $[0,2 \pi)^{2}$, and moreover the restriction of this trajectory to at $+\varphi_{1} \bmod 2 \pi=0$ is dense in $\{0\} \times[0,2 \pi)$.

If $|D|<|C|$, then we argue that $f$ is infinitely often negative, and hence has infinitely many zeros. Indeed, $|D|<|C|$ entails the existence of a non-trivial interval $I \subseteq[0,2 \pi)$ such that

$$
t \bmod 2 \pi \in I \Rightarrow C \cos \left(b t+\varphi_{2}\right)+D<0 .
$$

What is more, we can in fact find $\epsilon>0$ and a subinterval $I^{\prime} \subseteq I$ such that

$$
t \bmod 2 \pi \in I^{\prime} \Rightarrow C \cos \left(b t+\varphi_{2}\right)+D<-\epsilon .
$$

Thus, by density, $1-\cos \left(a t+\varphi_{1}\right)=0$ and $C \cos \left(b t+\varphi_{2}\right)+D<$ $-\epsilon$ will infinitely often hold simultaneously. Then just take $t$ large enough to ensure, say, $\left|e^{-r_{2} t} F(t)\right|<\epsilon / 2$ at these infinitely many points, and the claim follows.

Thus, suppose now $|C|=|D|$. Replacing $\varphi_{2}$ by $\varphi_{2}+\pi$ if necessary, we can write the function as:

$$
\begin{aligned}
f(t)= & 1-\cos \left(a t+\varphi_{1}\right) \\
& +D e^{-r_{1} t}\left(1-\cos \left(b t+\varphi_{2}\right)\right)+e^{-\left(r_{1}+r_{2}\right) t} F(t) .
\end{aligned}
$$

As $a, b$ are linearly independent, for all $t$ large enough, $1-$ $\cos \left(a t+\varphi_{1}\right)$ and $1-\cos \left(b t+\varphi_{2}\right)$ cannot simultaneously be 'too small'. More precisely, by Lemma2 2 there exist effective constants $E, T, N>0$ such that for all $t \geq T$, we have

$$
1-\cos \left(a t+\varphi_{1}\right)>E / t^{N} \text { or } 1-\cos \left(b t+\varphi_{2}\right)>E / t^{N} .
$$

Now, if $D<0$, it is easy to show that $f$ has infinitely many zeros. Indeed, consider the times $t$ where the dominant term $1-\cos (a t+$ $\left.\varphi_{1}\right)$ vanishes. For all large enough such $t$, since $t^{-N}$ shrinks more slowly than $e^{-r_{2} t}$, we will have

$$
\begin{aligned}
f(t) & =e^{-r_{1} t} D\left(1-\cos \left(b t+\varphi_{2}\right)\right)+e^{-\left(r_{1}+r_{2}\right) t} F(t) \\
& <e^{-r_{1} t}\left(E D t^{-N}+e^{-r_{2} t} F(t)\right) \\
& \leq e^{-r_{1} t} \frac{1}{2} E D t^{-N} \\
& <0
\end{aligned}
$$

so $f$ has infinitely many zeros. Similarly, if $D>0$, we can show that $f$ is ultimately positive. Indeed, for all $t$ large enough, we have

$$
\begin{aligned}
f(t) & \geq e^{-r_{1} t} D\left(1-\cos \left(b t+\varphi_{2}\right)\right)+e^{-\left(r_{1}+r_{2}\right) t} F(t) \\
& >e^{-r_{1} t} D E t^{-N}+e^{-\left(r_{1}+r_{2}\right) t} F(t) \\
& >0
\end{aligned}
$$

or

$$
\begin{aligned}
f(t) & \geq 1-\cos \left(a t+\varphi_{1}\right)+e^{-\left(r_{1}+r_{2}\right) t} F(t) \\
& >E t^{-N}+e^{-\left(r_{1}+r_{2}\right) t} F(t) \\
& >0 .
\end{aligned}
$$

Therefore, $f$ has only finitely many zeros.

Case II. Now suppose $a, b$ are linearly dependent. By the premise of the Lemma, the order of $F$ is at most 2 (in fact, at most 1 if $D \neq 0$ ). However, by Theorem 8 the claim follows immediately for all cases in which the characteristic roots of $F$ are all real or complex but with frequencies linearly dependent on $a$. Thus, the only remaining case to consider is the function

$$
\begin{aligned}
f(t)= & 1-\cos \left(a t+\varphi_{1}\right) \\
& +e^{-r_{1} t} C \cos \left(b t+\varphi_{2}\right)+e^{-\left(r_{1}+r_{2}\right) t} H \cos \left(c t+\varphi_{3}\right),
\end{aligned}
$$

where $H, c \in \mathbb{R} \cap \mathbb{A}, c>0$ and $a / c \notin \mathbb{Q}$.

As explained at the beginning of the proof of Lemma 10, due to the linear dependence of $a, b$ over $\mathbb{Q}$, when $1-\cos \left(a t+\varphi_{1}\right)=0$, there are only finitely many possibilities for the value of $C \cos (b t+$ $\varphi_{2}$ ), each algebraic, effectively computable and occurring periodically. If at least one of these values is non-positive, then by the linear independence of $a, c$ over $\mathbb{Q}$, we will simultaneously have $1-\cos \left(a t+\varphi_{1}\right)=0, C \cos \left(b t+\varphi_{2}\right) \leq 0$ and $H \cos \left(c t+\varphi_{3}\right)<0$ infinitely often, which yields $f(t)<0$ infinitely often and entails the existence of infinitely many zeros. On the other hand, if at the critical points $1-\cos \left(a t+\varphi_{1}\right)=0$ we always have $C \cos \left(b t+\varphi_{2}\right)>0$, then by Lemma 10 , we have

$$
1-\cos \left(a t+\varphi_{1}\right)+e^{-r_{1} t} C \cos \left(b t+\varphi_{2}\right)=\Omega\left(e^{-r_{1} t}\right),
$$

whereas obviously

$$
\left|e^{-\left(r_{1}+r_{2}\right) t} H \cos \left(c t+\varphi_{3}\right)\right|=\mathcal{O}\left(e^{-\left(r_{1}+r_{2}\right) t}\right) .
$$

If follows that $f$ is ultimately positive and hence has only finitely many zeros.

Lemma 12. Let $A, B, a, b, c, r$ be real algebraic numbers such that $a, b, c, r>0, A, B \neq 0$. Let also $\varphi_{1}, \varphi_{2}, \varphi_{3} \in \mathbb{R}$ be such that $e^{i \varphi_{1}}, e^{i \varphi_{2}}, e^{i \varphi_{3}} \in \mathbb{A}$. Define the exponential polynomial $f$ by

$f(t)=1-\cos \left(c t+\varphi_{3}\right)+e^{-r t}\left(A \cos \left(a t+\varphi_{1}\right)+B \cos \left(b t+\varphi_{2}\right)\right)$.

Then it is decidable whether $f$ has infinitely many zeros. 
Proof. We argue the function is infinitely often positive and infinitely often negative by looking at the values of $t$ for which the dominant term $1-\cos \left(c t+\varphi_{3}\right)$ vanishes. This happens precisely at the times $t=-\left(\varphi_{3}+2 k \pi\right) / c$ for $k \in \mathbb{Z}$, giving rise to a discrete restriction of $f$ :

$$
\begin{aligned}
& g(k) \stackrel{\text { def }}{=} e^{r \varphi_{3}}\left(e^{2 \pi r}\right)^{k}( A \cos \left(k \frac{2 \pi a}{c}-\frac{a \varphi_{3}}{c}+\varphi_{1}\right)+ \\
&\left.B \cos \left(k \frac{2 \pi b}{c}-\frac{b \varphi_{3}}{c}+\varphi_{2}\right)\right) .
\end{aligned}
$$

This is a linear recurrence sequence over $\mathbb{R}$ of order 4 , with characteristic roots $e^{2 \pi(r \pm i a / c)}$ and $e^{2 \pi(r \pm i b / c)}$. In particular, it has no real dominant characteristic root. It is well-known that real-valued linear recurrence sequences with no dominant real characteristic root are infinitely often positive and infinitely often negative: see for example (Győri and Ladas 1991, Theorem 7.1.1). Therefore, by continuity, $f$ must have infinitely many zeros.

Lemma 13. Let $A, B, a, b, r$ be real algebraic numbers such that $a, b, r>0, A \neq 0$. Let also $\varphi_{1}, \varphi_{2}, \varphi_{3} \in \mathbb{R}$ be such that $e^{i \varphi_{1}}, e^{i \varphi_{2}}, e^{i \varphi_{3}} \in \mathbb{A}$. Define the exponential polynomial $f$ by

$f(t)=1-\cos \left(a t+\varphi_{1}\right)+e^{-r t}\left(A t \cos \left(b t+\varphi_{2}\right)+B \cos \left(b t+\varphi_{3}\right)\right)$.

Then it is decidable whether $f$ has infinitely many zeros.

Proof. If $a / b \in \mathbb{Q}$, then the claim follows immediately from Theorem 8 If $a / b \notin \mathbb{Q}$, then by Lemma 4 it will happen infinitely often that $1-\cos \left(a t+\varphi_{1}\right)=0$ and $A t \cos \left(b t+\varphi_{2}\right)<-|A| t / 2$. Then clearly $f(t)<0$ infinitely often. Since $f(t)>0$ infinitely often as well, due to the non-negative dominant term $1-\cos (a t+$ $\left.\varphi_{1}\right)$, it follows that $f$ has infinitely many zeros.

\subsection{Two dominant oscillations}

Lemma 14. Let $A, B, C, a, b, r$ be real algebraic numbers such that $a, b, r>0, a \neq b$ and $A, B, C \neq 0$. Let also $\varphi_{1}, \varphi_{2} \in \mathbb{R}$ be such that $e^{i \varphi_{1}}, e^{i \varphi_{2}} \in \mathbb{A}$. Define the exponential polynomial $f$ by

$$
f(t)=A \cos \left(a t+\varphi_{1}\right)+B \cos \left(b t+\varphi_{2}\right)+C+e^{-r t} F(t) .
$$

where $F$ is an exponential polynomial whose dominant characteristic roots are purely imaginary. Suppose also f has order at most 8. It is decidable whether $f$ has infinitely many zeros.

Proof. If the frequencies $a, b$ of the dominant term's oscillations are linearly independent over $\mathbb{Q}$, then the claim follows immediately by Theorem 7 Therefore, assume $n a-m b=0$ for some $n, m \in \mathbb{N}^{+}$. Notice that $a \neq b$ guarantees $n \neq m$. We perform the change of variable $t \rightarrow t m / a$, so that:

$$
f(t)=A \cos \left(m t+\varphi_{1}\right)+B \cos \left(n t+\varphi_{2}\right)+C+e^{-r m t / a} F(t m / a) .
$$

Using the standard trigonometric identities, we express the dominant term as a polynomial in $\sin (t), \cos (t)$ :

$$
f(t)=P(\sin (t), \cos (t))+e^{-r m t / a} F(t m / a),
$$

where $P \in(\mathbb{R} \cap \mathbb{A})[x, y]$ has effectively computable coefficients. It is clear that the dominant term is periodic. It is immediate from the definition of exponential polynomials and the premise of the Lemma that $F(t m / a) \stackrel{\text { def }}{=} F_{2}(t)$ is an exponential polynomial in $t$, of the same order as $F(t)$, also with purely imaginary dominant characteristic roots. Let $\alpha(t) \stackrel{\text { def }}{=} P(\sin (t), \cos (t)), r_{2} \stackrel{\text { def }}{=} \mathrm{rm} / a>$ 0 and $\beta(t) \stackrel{\text { def }}{=} e^{-r m t / a} F(t m / a)=e^{-r_{2} t} F_{2}(t)$.
We are now interested in the extrema of $P(\sin (t), \cos (t))$. Let

$$
\begin{gathered}
M_{1} \stackrel{\text { def }}{=} \min _{x^{2}+y^{2}=1} P(x, y)=\min _{t \geq 0} \alpha(t), \\
M_{2} \stackrel{\text { def }}{=} \max _{x^{2}+y^{2}=1} P(x, y)=\max _{t \geq 0} \alpha(t) .
\end{gathered}
$$

We can construct defining formulas $\phi_{1}(u), \phi_{2}(u)$ in the first-order language $\mathcal{L}$ of real closed fields for $M_{1}, M_{2}$, so that each $\phi_{j}(u)$ holds precisely for the valuation $u=M_{j}$. Then performing quantifier elimination on these formulas using Renegar's algorithm (Renegar 1992), we convert $\phi_{1}, \phi_{2}$ into the form

$$
\phi_{j}(u) \equiv \bigvee_{l} \bigwedge_{k} P_{l, k}(u) \sim_{l, k} 0,
$$

where $P_{l, k}$ are polynomials with integer coefficients and each $\sim_{l, k}$ is either $<$ or $=$. Now $\phi_{j}(u)$ must have a satisfiable disjunct. Using the decidability of the theory $T h(\mathbb{R})$, we can readily identify this disjunct. Moreover, since $\phi_{j}(u)$ has a unique satisfying valuation, namely $u=M_{j}$, this disjunct must contain at least one equality predicate. It follows immediately that $M_{1}, M_{2}$ are algebraic. Moreover, we can effectively compute from $\phi_{j}(u)$ a representation for $M_{j}$ consisting of its minimal polynomial and a sufficiently accurate rational approximation to distinguish $M_{j}$ from its Galois conjugates. By an analogous argument, the pairs $(\sin (t), \cos (t))$ at which $P(\sin (t), \cos (t))$ achieves the extrema $M_{1}, M_{2}$ are also algebraic and effectively computable.

We now perform a case analysis on the signs of $M_{1}$ and $M_{2}$.

- First, if $0<M_{1} \leq M_{2}$, then $f(t)$ cannot have infinitely many zeros: if $t$ is large enough to ensure $|\beta(t)|<M_{1}$, we have $f(t)>0$.

- Second, if $M_{1} \leq M_{2}<0$, then by the same reasoning, the function will ultimately be strictly negative.

- Third, if $M_{1}<0<M_{2}$, then $f$ oscillates around 0: for all $t$ such that $\alpha(t)=M_{1}<0$ and large enough to ensure $|\beta(t)|<\left|M_{1}\right|$, we will have $f(t)<0$, and similarly, for large enough $t$ such that $\alpha(t)=M_{2}>0$, we will have $f(t)>0$, so the function must have infinitely many zeros.

- Next, we argue that the case $M_{1}=M_{2}=0$ is impossible. Indeed, if $M_{1}=M_{2}=0$, then $\alpha(t)=P(\sin (t), \cos (t))$ is identically zero, and the same holds for all derivatives of $\alpha(t)$. Thus, from $\alpha^{\prime}(t) \equiv \alpha^{\prime \prime \prime}(t) \equiv 0$, we have

$$
\begin{aligned}
& 0 \equiv-A m \sin \left(m t+\varphi_{1}\right)-B n \sin \left(n t+\varphi_{2}\right), \\
& 0 \equiv A m^{3} \sin \left(m t+\varphi_{1}\right)+B n^{3} \sin \left(n t+\varphi_{2}\right)
\end{aligned}
$$

Multiplying the first identity through by $\mathrm{m}^{2}$ and summing, we have

$$
B n \sin \left(n t+\varphi_{2}\right)\left(n^{2}-m^{2}\right) \equiv 0 .
$$

By the premise of the Lemma, $B \neq 0$, so $n(n-m)(n+m)=$ 0 , which is a contradiction.

- Finally, only the symmetric cases $M_{1}<M_{2}=0$ and $0=$ $M_{1}<M_{2}$ remain. Without loss of generality, by replacing $f$ by $-f$ if necessary, we need only consider the case $0=M_{1}<$ $M_{2}$.

Thus, assume $0=M_{1}<M_{2}$. We now move our attention to the possible forms of $F_{2}$. Since $f$ has order at most 8 , it follows that $F_{2}$ has order at most 3 . Thus, there are three possibilities for the set of dominant characteristic roots of $F_{2}:\{0\},\{ \pm i c\}$, or $\{0, \pm i c\}$, for some positive $c \in \mathbb{R} \cap \mathbb{A}$. We consider each of these cases in turn.

First, if $F_{2}$ only has the real dominant eigenvalue 0 , then $F_{2}$ is ultimately positive or ultimately negative, depending on the sign of 
the most significant term of $F_{2}$. Ultimate positivity of $F_{2}$ entails ultimate positivity of $f$ as well, since $P(\sin (t), \cos (t)) \geq 0$ everywhere, whereas an ultimately negative $F_{2}$ makes $f$ change sign infinitely often.

Second, assume the dominant characteristic roots of $F_{2}$ are $\{ \pm i c\}$, so that

$$
f(t)=P(\sin (t), \cos (t))+e^{-r_{2} t}\left(D \cos \left(c t+\varphi_{3}\right)+E e^{-r_{3} t}\right)
$$

for some $r_{3}>0$ and $\varphi_{3} \in \mathbb{R}$ such that $e^{i \varphi_{3}} \in \mathbb{A}$. Without loss of generality, we can assume $c \notin \mathbb{Q}$, since otherwise, we are done by Theorem 8 But by Lemma 4 it will happen infinitely often that $P(\sin (t), \cos (t))=0$ and $D \cos \left(c t+\varphi_{3}\right)<-|D| / 2$, say. For large enough such $t,\left|E e^{-\left(r_{2}+r_{3}\right) t}\right|<|D| / 4$, so we conclude that $f$ is infinitely often negative, and hence has infinitely many zeros.

Third, assume the dominant characteristic roots of $F_{2}(t)$ are $\{0, \pm i c\}$, so that

$$
f(t)=P(\sin (t), \cos (t))+e^{-r_{2} t}\left(D \cos \left(c t+\varphi_{3}\right)+E\right) .
$$

We again assume $c \notin \mathbb{Q}$, since otherwise the claim follows from Theorem 8 Let $M_{3} \stackrel{\text { def }}{=} E-|D|=\min _{t \geq 0} F_{2}(t)$. If $M_{3}>0$, then $f(t)$ clearly has no zeros. If $M_{3}<0$, then there exists a non-trivial interval $I \subseteq[0,2 \pi)$ such that if $c t+\varphi_{3} \bmod 2 \pi \in$ $I$, then $F_{2}(t)<0$. Since $c \notin \mathbb{Q}$, Lemma 4 guarantees that $F_{2}(t)<0=P(\sin (t), \cos (t))$ happens infinitely often, so $f$ must have infinitely many zeros. Finally, if $M_{3}=0$, we argue that $f$ is ultimately positive. Indeed, since $P(\sin (t), \cos (t))$ and $F_{2}(t)$ are both non-negative everywhere, $f(t)=0$ can only happen if $P(\sin (t), \cos (t))=D \cos \left(c t+\varphi_{3}\right)+E=0$. This, however, would entail $e^{i t} \in \mathbb{A}$ and $e^{i c t} \in \mathbb{A}$, which contradicts the GelfondSchneider Theorem, since $c \notin \mathbb{Q}$. Thus, we conclude $f$ has no zeros.

\subsection{Three dominant oscillations}

Lemma 15. Let $A, B, C, a, b, c$ be real algebraic numbers such that $a, b, c>0$ and $A, B, C \neq 0$. Let also $\varphi_{1}, \varphi_{2}, \varphi_{3} \in \mathbb{R}$ be such that $e^{i \varphi_{1}}, e^{i \varphi_{2}}, e^{i \varphi_{3}} \in \mathbb{A}$. Define the exponential polynomial $f$ by

$f(t)=A \cos \left(a t+\varphi_{1}\right)+B \cos \left(b t+\varphi_{2}\right)+C \cos \left(c t+\varphi_{3}\right)+D$.

It is decidable whether $f$ has infinitely many zeros.

Proof. The argument consists of three cases, depending on the linear dependencies over $\mathbb{Q}$ satisfied by $a, b$ and $c$.

Case $I$. First, if $a, b, c$ are linearly independent over $\mathbb{Q}$, then the claim follows directly from Theorem 7

Case II. Second, suppose that $a, b, c$ are all rational multiples of one another:

$$
b=\frac{n}{m} a, c=\frac{k}{l} a \text { where } n, m, k, l \in \mathbb{N}^{+} .
$$

We make the change of variable $t \rightarrow t m l$ to obtain:

$$
\begin{aligned}
f(t)= & A \cos \left((a t) m l+\varphi_{1}\right)+B \cos \left((a t) n l+\varphi_{2}\right) \\
& +C \cos \left((a t) k m+\varphi_{3}\right)+D \\
= & P(\sin (a t), \cos (a t)),
\end{aligned}
$$

where $P \in \mathbb{A}[x, y]$ is a polynomial obtained using the standard trigonometric identities. It is now clear that $f$ is periodic, so it has either no zeros or infinitely many zeros. Let

$$
\begin{gathered}
M_{1} \stackrel{\text { def }}{=} \min _{x^{2}+y^{2}=1} P(x, y)=\min _{t \geq 0} f(t), \\
M_{2} \stackrel{\text { def }}{=} \max _{x^{2}+y^{2}=1} P(x, y)=\max _{t \geq 0} f(t) .
\end{gathered}
$$

Using the same reasoning as in Lemma 14 we see that $M_{1}, M_{2}$ are algebraic and effectively computable: simply construct defining formulas in the first-order language $\mathcal{L}$ of real closed fields, and then perform quantifier elimination using Renegar's algorithm (Renegar 1992). Then $f$ clearly has infinitely many zeros if and only if $M_{1} \leq 0 \leq M_{2}$.

Case III. Finally, suppose that $a, b, c$ span a $\mathbb{Q}$-vector space of dimension 2 , so that $a, b, c$ satisfy a single linear dependence $a m+b n+c p=0$ where $m, n, p \in \mathbb{Z}$ are coprime. At most one of the ratios $a / b, a / c$ and $b / c$ is rational (otherwise we have $\operatorname{dim} \operatorname{span}\{a, b, c\}=1)$, so assume without loss of generality that $a / c \notin \mathbb{Q}$ and $b / c \notin \mathbb{Q}$.

Define the set

$$
\begin{aligned}
\mathbb{T} & \stackrel{\text { def }}{=}\left\{x \in[0,2 \pi)^{3} \mid \forall u \in \mathbb{Z}^{3} \cdot u \cdot(a, b, c) \in 2 \pi \mathbb{Z} \Rightarrow u \cdot x \in 2 \pi \mathbb{Z}\right\} \\
& =\left\{\left(x_{1}, x_{2}, x_{3}\right) \in[0,2 \pi)^{3} \mid m x_{1}+n x_{2}+p x_{3} \in 2 \pi \mathbb{Z}\right\}
\end{aligned}
$$

Notice that if $m x_{1}+n x_{2}+p x_{3}=2 k \pi$ for some $x_{1}, x_{2}, x_{3}$, then $k \leq|m|+|n|+|p|$, so $\mathbb{T}$ partitions naturally into finitely many subsets: $\mathbb{T}=\bigcup_{k=1}^{N} \mathbb{T}_{k}$, where

$$
\mathbb{T}_{k} \stackrel{\text { def }}{=}\left\{\left(x_{1}, x_{2}, x_{3}\right) \in[0,2 \pi)^{3} \mid m x_{1}+n x_{2}+p x_{3}=2 k \pi\right\} .
$$

Consider the trajectory $h(t) \stackrel{\text { def }}{=}\{(a t, b t, c t) \bmod 2 \pi \mid t \geq 0\}$. Define also the sets $R \stackrel{\text { def }}{=}\{h(2 k \pi) \mid k \in \mathbb{N}\}$ and $H \stackrel{\text { def }}{=}\{h(t) \mid t \geq$ $0\}$. Because of the linear dependence satisfied by $a, b, c$, it is easy to see that $R \subseteq H \subseteq \mathbb{T}$. By Kronecker's Theorem, $R$ is a dense subset of $\mathbb{T}$, so clearly $H$ must be a dense subset of $\mathbb{T}$ as well.

Now define the function

$$
\begin{aligned}
F\left(x_{1}, x_{2}, x_{3}\right) \stackrel{\text { def }}{=} & A \cos \left(x_{1}+\varphi_{1}\right)+B \cos \left(x_{2}+\varphi_{2}\right) \\
& +C \cos \left(x_{3}+\varphi_{3}\right)+D
\end{aligned}
$$

so that the image of $f$ is exactly $\left\{F\left(x_{1}, x_{2}, x_{3}\right) \mid\left(x_{1}, x_{2}, x_{3}\right) \in\right.$ $H\}$. Let also the extrema of $F$ over $\mathbb{T}$ be:

$$
\begin{aligned}
& M_{1} \stackrel{\text { def }}{=} \min _{\mathbb{T}} F\left(x_{1}, x_{2}, x_{3}\right), \\
& M_{2} \stackrel{\text { def }}{=} \max _{\mathbb{T}} F\left(x_{1}, x_{2}, x_{3}\right) .
\end{aligned}
$$

Both of these values are algebraic and can be computed using quantifier elimination in the first-order language $\mathcal{L}$ of the real numbers: just use separate variables for $\cos \left(x_{j}\right), \sin \left(x_{j}\right)$ and apply the standard trigonometric identities to convert the linear dependence on $x_{1}, x_{2}, x_{3}$ into a polynomial dependence between $\cos \left(x_{j}\right), \sin \left(x_{j}\right)$.

Now, by the density of $H$ in $\mathbb{T}$, if $M_{1}<0<M_{2}$, then $f$ must clearly be infinitely often positive and infinitely often negative, so it must have infinitely many zeros. The case $M_{1}<0=M_{2}$ is symmetric to $0=M_{1}<M_{2}$ (just replace $f$ and $F$ by $-f$ and $-F$, respectively), so without loss generality, we can assume $0=M_{1}<M_{2}$. In this case, we argue that $f$ has no zeros, that is, even though $F$ vanishes on some points in $\mathbb{T}$, none of these points appear in the dense subset $H$. Indeed, consider the set

$$
\begin{gathered}
Z \stackrel{\text { def }}{=}\left\{\left(\cos \left(x_{1}\right), \sin \left(x_{1}\right), \ldots, \cos \left(x_{3}\right), \sin \left(x_{3}\right)\right) \mid\right. \\
\left.\left(x_{1}, x_{2}, x_{3}\right) \in \mathbb{T}, F\left(x_{1}, x_{2}, x_{3}\right)=0\right\} .
\end{gathered}
$$

Note that $Z$ is clearly semi-algebraic, as one can directly write a defining formula in $\mathcal{L}$ from $F\left(x_{1}, x_{2}, x_{3}\right)=0$ and $m x_{1}+$ $n x_{2}+p x_{3} \in 2 \pi \mathbb{Z}$. Moreover, by the Zero-Dimensionality Lemma (Ouaknine and Worrell 2014, Lemma 10), the function $F\left(x_{1}, x_{2}, x_{3}\right)$ achieves its minimum $M_{1}=0$ at only finitely many points in $\mathbb{T}_{k}$, for each $k$. Since $\mathbb{T}$ is the union of finitely many $\mathbb{T}_{k}$, we immediately have that $Z$ is finite. By the Tarski-Seidenberg Theorem, projecting $Z$ to any fixed component will also give a finite, semialgebraic subset of $\mathbb{R}$, that is, a finite subset of $\mathbb{A}$. Thus, we have shown that if $F\left(x_{1}, x_{2}, x_{3}\right)=0$, then $e^{i x_{j}} \in \mathbb{A}$ for all $j=1,2,3$. 
Now if $f(t)=0$ for some $t \geq 0$, then we must have $e^{a t i}, e^{c t i} \in \mathbb{A}$, which by the Gelfond-Schneider Theorem entails $a / c \in \mathbb{Q}$, a contradiction.

\subsection{One repeated oscillation}

Lemma 16. Let $A, B, C, D, a, r$ be real algebraic numbers such that $a, r>0$ and $A \neq 0$. Let also $\varphi_{1}, \varphi_{2} \in \mathbb{R}$ be such that $e^{i \varphi_{1}}, e^{i \varphi_{2}} \in \mathbb{A}$. Define the exponential polynomial $f$ by

$f(t)=t\left(A \cos \left(a t+\varphi_{1}\right)+B\right)+\left(C \cos \left(a t+\varphi_{2}\right)+D\right)+e^{-r t} F(t)$

where $F$ is an exponential polynomial with purely imaginary dominant characteristic roots. Suppose also that $f$ has order at most 8. It is decidable whether $f$ has infinitely many zeros.

Proof. Since $f$ has order no greater than 8 , it follows that $F$ has order at most 2. Therefore, $F(t)$ must be of the form $E \cos \left(b t+\varphi_{3}\right)$ for some $E, b \in \mathbb{R} \cap \mathbb{A}, b>0$, such that $a / b \notin \mathbb{Q}$, and some $\varphi_{3}$ such that $e^{i \varphi_{3}} \in \mathbb{A}$, since otherwise the imaginary parts of the characteristic roots of $f$ are pairwise linearly dependent over $\mathbb{Q}$, so our claim is proven immediately by Theorem 8

Consider first the magnitudes of $A$ and $B$. If $|A|>|B|$, then the term $t A \cos \left(a t+\varphi_{1}\right)$ makes $f$ change sign infinitely often, whereas if $|B|>|A|$, then for $t$ large enough, the term $t B$ makes $f$ ultimately positive or ultimately negative, depending on the sign of $B$. Thus, we can assume $|A|=|B|$. Dividing $f$ by $B$, and replacing $\varphi_{1}$ by $\varphi_{1}+\pi$ if necessary, we can assume the function has the form:

$$
\begin{aligned}
f(t)= & t\left(1-\cos \left(a t+\varphi_{1}\right)\right) \\
& +\left(C \cos \left(a t+\varphi_{2}\right)+D\right)+e^{-r t} E \cos \left(b t+\varphi_{3}\right) .
\end{aligned}
$$

Considering the dominant term, it is clear that $f$ is infinitely often positive. Let $\alpha(t) \stackrel{\text { def }}{=} t\left(1-\cos \left(a t+\varphi_{1}\right)\right), \beta(t) \stackrel{\text { def }}{=} C \cos (a t+$ $\left.\varphi_{2}\right)+D$ and $\gamma(t) \stackrel{\text { def }}{=} e^{-r t} E \cos \left(b t+\varphi_{3}\right)$.

We now focus on the sign of the term $\beta(t)$ at the positive critical times $t_{j} \stackrel{\text { def }}{=}-\varphi_{1} / a+2 j \pi / a(j \in \mathbb{Z})$ when $1-\cos (a t+$ $\left.\varphi_{1}\right)$ vanishes. Notice that $\beta\left(t_{j}\right)=C \cos \left(\varphi_{2}-\varphi_{1}\right)+D \stackrel{\text { def }}{=}$ $M$ is independent of $j$. First, if $M<0$, then for all $t_{j}$ large enough, $f\left(t_{j}\right)<0$, so the function must have infinitely many zeros. Second, if $M=0$, then by the linear independence of $a, b$ and Lemma 4 we have $\alpha\left(t_{j}\right)=\beta\left(t_{j}\right)=0>\gamma\left(t_{j}\right)$ for infinitely many $t_{j}$, so we can conclude $f$ has infinitely many zeros.

Finally, suppose $M>0$. We will prove that $f$ is ultimately positive. For each $t_{j}$, define the critical region $\left[t_{j}-\delta_{j}, t_{j}+\delta_{j}\right]$, given by

$$
\delta_{j} \stackrel{\text { def }}{=} \frac{2 \sqrt{|C|+|D|}}{a \sqrt{t_{j-1}}} .
$$

From here onwards, we only consider $t$ large enough for any two adjacent critical regions to be disjoint. The argument consists of two parts: first we show $f(t)>0$ for all large enough $t$ outside all critical regions, and then we show $f(t)>0$ for large enough $t$ in a critical region.

Suppose $t$ is outside all critical regions and let $j$ minimise $\left|t-t_{j}\right|$. Since the distance between critical points is $2 \pi / a$ by construction, we have $a\left|t-t_{j}\right| \leq \pi$. Therefore,

$$
\frac{\left|a\left(t-t_{j}\right)\right|^{2}}{2} \leq 1-\cos \left(a t-a t_{j}\right)=1-\cos \left(a t+\varphi_{1}\right) .
$$

On the other hand, we have the following chain of inequalities:

$$
\begin{aligned}
& \frac{\left|a\left(t-t_{j}\right)\right|^{2}}{2} \\
> & \left\{\left|t-t_{j}\right|>\delta_{j}\right\} \\
& \frac{\left(a \delta_{j}\right)^{2}}{2} \\
= & \left\{\text { definition of } \delta_{j}\right\} \\
& \frac{2(|C|+|D|)}{t_{j-1}} \\
> & \left\{\text { by } t>t_{j-1}\right\} \\
& \frac{2(|C|+|D|)}{t} \\
\geq & \{\text { triangle inequality and }|\cos (x)| \leq 1\} \\
& \frac{|C|+|D|}{t}+\frac{\left|C \cos \left(a t+\varphi_{2}\right)+D\right|}{t} .
\end{aligned}
$$

Combining, we have

$$
\begin{aligned}
\alpha(t)+\beta(t) & \geq \alpha(t)-|\beta(t)| \\
& =t\left(1-\cos \left(a t+\varphi_{1}\right)\right)-\left|C \cos \left(a t+\varphi_{2}\right)+D\right| \\
& \geq|C|+|D| .
\end{aligned}
$$

Thus, if $t$ is large enough to ensure $|\gamma(t)|<|C|+|D|$, we have $f(t)>0$ outside critical regions.

For the second part of the argument, we consider $t$ in critical regions. Notice that the values of $\beta(t)$ on $\left[t_{j}-\delta_{j}, t_{j}+\delta_{j}\right]$ are independent of the choice of $t_{j}$. Moreover, we have $\beta\left(t_{j}\right)=M>$ 0 , so there exists some $\epsilon>0$ such that for all $t \in\left[t_{j}-\epsilon, t_{j}+\epsilon\right]$, we have $\beta(t) \geq M / 2$, say. Now for any critical point $t_{j}$ chosen large enough, we will have $\left[t_{j}-\delta_{j}, t_{j}+\delta_{j}\right] \subseteq\left[t_{j}-\epsilon, t_{j}+\epsilon\right]$, so $\beta(t)>$ $M / 2$ on the entire critical region. Let also $t_{j}$ be large enough so that for any $t$ in the critical region, we have $|\gamma(t)|<M / 2$. Then we have $f(t)=\alpha(t)+\beta(t)+\gamma(t) \geq \beta(t)-|\gamma(t)|>0$, completing the claim.

\section{Hardness at Order 9}

Diophantine approximation is a branch of number theory concerned with approximating real numbers by rationals. A central role is played in this theory by the notion of continued fraction expansion, which allows to compute a sequence of rational approximations to a given real number that is optimal in a certain well-defined sense. For our purposes it suffices to note that the behaviour of the continued fraction expansion of a real number $a$ is closely related to the following two constants associated with $a$. The Lagrange constant (or homogeneous Diophantine approximation constant) of a is defined by

$L_{\infty}(a)=\inf \left\{c:\left|a-\frac{n}{m}\right|<\frac{c}{m^{2}}\right.$ for infinitely many $\left.m, n \in \mathbb{Z}\right\}$.

By definition $L_{\infty}(a)$ is a non-negative real number.

A real number $a$ is called badly approximable if $L_{\infty}(a)>$ 0 . The badly approximable numbers are precisely those whose continued fraction expansions have bounded partial quotients.

Khinchin showed in 1926 that almost all real numbers (in the measure-theoretic sense) have Lagrange constant equal to zero. However, information on the Lagrange constants of specific numbers or classes of numbers has proven to be elusive. In particular, concerning algebraic numbers, Guy (Guy 2004) asks

Is there an algebraic number of degree greater than two whose simple continued fraction expansion has unbounded 
partial quotients? Does every such number have unbounded partial quotients?

The above question can equivalently be formulated in terms of whether any algebraic number of degree greater than two has stricly positive Lagrange constant or whether all such numbers have Lagrange constant 0 .

Recall that a real number $a$ is computable if there is an algorithm which, given any rational $\varepsilon>0$ as input, returns a rational $q$ such that $|q-x|<\varepsilon$. We can now state the main result of the section.

In this section, we will show that a decision procedure for the Infinite Zeros Problem would yield the computability of $L_{\infty}(a)$ for all $a \in \mathbb{R} \cap \mathbb{A}$.

Fix positive $a \in \mathbb{R} \cap \mathbb{A}, c \in \mathbb{Q}$ and define the functions:

$$
\begin{aligned}
f_{1}(t) & \stackrel{\text { def }}{=} e^{t}(1-\cos (t))+t(1-\cos (a t))-c \sin (a t), \\
f_{2}(t) & \stackrel{\text { def }}{=} e^{t}(1-\cos (t))+t(1-\cos (a t))+c \sin (a t), \\
f(t) & \stackrel{\text { def }}{=} e^{t}(1-\cos (t))+t(1-\cos (a t))-c|\sin (a t)| \\
& =\min \left\{f_{1}(t), f_{2}(t)\right\} .
\end{aligned}
$$

It is easy to see that $f_{1}$ and $f_{2}$ are exponential polynomials of order 9, with six characteristic roots: three simple ( 1 and $1 \pm i$ ) and three repeated $(0$ and $\pm a i)$. Thus, the problem of determining whether $f_{j}$ has infinitely many zeros is an instance of the Infinite Zeros Problem. Moreover, it is easy to check that $f$ has infinitely many zeros if and only if at least one of $f_{1}$ and $f_{2}$ has infinitely many zeros.

We will first state two lemmas which show a connection between the existence of infinitely many zeros of $f$ and the Lagrange constant of $a$. We defer the proofs to Appendix A

Lemma 17. Fix $a \in \mathbb{R} \cap \mathbb{A}$ and $\varepsilon, c \in \mathbb{Q}$ with $a, c>0$ and $\varepsilon \in(0,1)$. If $f(t)=0$ for infinitely many $t \geq 0$, then $L_{\infty}(a) \leq c / 2 \pi^{2}(1-\varepsilon)$.

Lemma 18. Fix $a \in \mathbb{R} \cap \mathbb{A}$ and $\varepsilon, c \in \mathbb{Q}$ with $a, c>0$ and $\varepsilon \in(0,1)$. If $L_{\infty}(a) \leq c(1-\varepsilon) / 2 \pi^{2}$, then $f(t)=0$ for infinitely many $t \geq 0$.

We now use the above lemmas to derive an algorithm to compute $L_{\infty}(a)$ using an oracle for the Infinite Zeros Problem, establishing our central hardness result:

Theorem 19. Fix a positive real algebraic number a. If the Infinite Zeros Problem is decidable for instances of order 9 , then $L_{\infty}(a)$ may be computed to within arbitrary precision.

Proof. Suppose we know $L_{\infty}(a) \in[p, q]$ for non-negative $p, q \in$ $\mathbb{Q}$. Choose $c \in \mathbb{Q}$ with $c>0$ and $\varepsilon \in \mathbb{Q}$ with $\varepsilon \in(0,1)$ such that

$$
p<\frac{c(1-\varepsilon)}{2 \pi^{2}}<\frac{c}{2 \pi^{2}(1-\varepsilon)}<q .
$$

Write $A \stackrel{\text { def }}{=} c(1-\varepsilon) / 2 \pi^{2}$ and $B \stackrel{\text { def }}{=} c / 2 \pi^{2}(1-\varepsilon)$. Use the oracle for the Infinite Zeros Problem to determine whether at least one of $f_{1}, f_{2}$ has infinitely many zeros. If this is the case, then $f$ also has infinitely many zeros, so by Lemma 17, $L_{\infty}(a) \leq B$ and we continue the approximation recursively on the interval $[p, B]$. If not, then $L(a) \geq A$ by Lemma 18 , so we continue on the interval $[A, q]$. Notice that in this procedure, one can choose $c, \varepsilon$ at each stage in such a way that the confidence interval shrinks by at least a fixed factor, whatever the outcome of the oracle invocations. It follows therefore that $L_{\infty}(a)$ can be approximated to within arbitrary precision.

Acknowledgements Ventsislav Chonev is supported by Austrian Science Fund (FWF) NFN Grant No S11407-N23 (RiSE/SHiNE), ERC Start grant (279307: Graph Games), and ERC Advanced
Grant (267989: QUAREM). Joël Ouaknine is supported by ERC grant AVS-ISS (648701). James Worrell is supported by EPSRC grant EP/N008197/1.

\section{References}

A. Baker. Transcendental number theory. Cambridge University Press, Cambridge, 1975.

P. C. Bell, J.-C. Delvenne, R. M. Jungers, and V. D. Blondel. The Continuous Skolem-Pisot Problem. Theoretical Computer Science, 411(40-42): 3625-3634, 2010. ISSN 0304-3975.

J. Berstel and M. Mignotte. Deux propriétés décidables des suites récurrentes linéaires. Bulletin de la Société Mathématique de France, 104:175-184, 1976.

H. Cohen. A Course in Computational Algebraic Number Theory. Springer, 1993.

A. O. Gelfond. On Hilbert's seventh problem. In Dokl. Akad. Nauk. SSSR, volume 2, pages 1-6, 1934.

A. O. Gelfond and I. Vinogradov. Sur le septieme probleme de Hilbert. Bull. Acad. Sci. URSS, pages 623-634, 1934.

R. Guy. Unsolved Problems in Number Theory. Springer, third edition, 2004.

I. Győri and G. Ladas. Oscillation Theory of Delay Differential Equations: with Applications. Oxford mathematical monographs. Oxford University Press, 1991. ISBN 9780198535829. URL https://books.google.co.uk/books?id=6CnvAAAAMAAJ

G. Hardy and E. Wright. An introduction to the theory of numbers. Oxford, 1:979, 1999.

A. Macintyre and A. J. Wilkie. On the decidability of the real exponential field. 1996.

D. Marker. Model Theory: An Introduction. Graduate Texts in Mathematics. Springer, 2002.

J. Ouaknine and J. Worrell. On the positivity problem for simple linear recurrence sequences. In Automata, Languages, and Programming, pages 318-329. Springer, 2014.

V. Pan. Optimal and nearly optimal algorithms for approximating polynomial zeros. Computers \& Mathematics with Applications, 31(12):97 138, 1996.

J. Renegar. On the computational complexity and geometry of the firstorder theory of the reals. part i: Introduction. preliminaries. the geometry of semi-algebraic sets. the decision problem for the existential theory of the reals. Journal of Symbolic Computation, 13(3):255 - 299, 1992. ISSN 0747-7171. doi: http://dx.doi.org/10.1016/S0747-7171(10) 80003-3.

T. Schneider. Transzendenzuntersuchungen periodischer Funktionen I. Transzendenz von Potenzen. Journal für die reine und angewandte Mathematik, 172:65-69, 1935a.

T. Schneider. Transzendenzuntersuchungen periodischer Funktionen II. Transzendenzeigenschaften elliptischer Funktionen. Journal für die reine und angewandte Mathematik, 172:70-74, 1935b.

T. Tao. Structure and randomness: pages from year one of a mathematical blog. American Mathematical Society, 2008.

A. Tarski. A decision method for elementary algebra and geometry. 1951.

A. J. Wilkie. Model completeness results for expansions of the ordered field of real numbers by restricted Pfaffian functions and the exponential function. Journal of the American Mathematical Society, pages 10511094, 1996.

\section{A. Proofs of Hardness Lemmas}

Throughout this section, let

$$
f(t) \stackrel{\text { def }}{=} e^{t}(1-\cos (t))+t(1-\cos (a t))-c|\sin (a t)| .
$$


Lemma 17. Fix $a \in \mathbb{R} \cap \mathbb{A}$ and $\varepsilon, c \in \mathbb{Q}$ with $a, c>0$ and $\varepsilon \in(0,1)$. If $f(t)=0$ for infinitely many $t \geq 0$, then $L_{\infty}(a) \leq c / 2 \pi^{2}(1-\varepsilon)$.

Proof. Suppose $f(t)=0$ for infinitely many $t$. Clearly, this also entails $f(t)=0$ for infinitely many $t \geq T$, for any particular threshold $T \geq 0$. (Indeed, $f(t)=\min \left\{f_{1}(t), f_{2}(t)\right\}$ for exponential polynomials $f_{1}$ and $f_{2}$ given at the beginning of Section 5 Thus, on any bounded interval, $f$ has no more zeros than $f_{1}$ and $f_{2}$ combined, i.e., only finitely many, by the analiticity of $f_{1}$ and $f_{2}$.) We will show that $T$ can be chosen in such a way that every zero of $f(t)$ on $[T, \infty)$ yields a pair $(n, m) \in \mathbb{N}^{2}$ which satisfies the inequality

$$
\left|a-\frac{n}{m}\right|<\frac{c}{2 \pi^{2} m^{2}(1-\varepsilon)} .
$$

This is sufficient, since infinitely many zeros of $f$ yield infinitely many solutions, and therefore witness $L_{\infty}(a) \leq c / 2 \pi^{2}(1-\varepsilon)$.

Thus, consider some $t$ such that $f(t)=0$ and $t \geq T$ for some threshold $T$ to be specified later. Let $t=2 \pi m+\delta_{1}$ and $a t=2 \pi n+\delta_{2}$, where $m, n \in \mathbb{N}$ and $\delta_{1}, \delta_{2} \in[-\pi, \pi)$. Then we have

$$
\left|a-\frac{n}{m}\right|=\frac{\left|\delta_{2}-a \delta_{1}\right|}{2 \pi m} .
$$

We will show that for $T$ large enough, $f(t)=0$ for $t \geq T$ allows us to bound $\left|\delta_{2}\right|$ and $\left|a \delta_{1}\right|$ separately from above and then apply the triangle inequality to bound $\left|\delta_{2}-a \delta_{1}\right|$.

First, choose $\varphi_{1}, \varphi_{2} \in(0,1)$ such that $1-\varphi_{2}>1-\varphi_{1}>1-\varepsilon$. Let $T$ be large enough for the following property to hold:

$$
\frac{t+\pi}{t-2 \pi} \leq \frac{1-\varphi_{2}}{1-\varphi_{1}} \text { for all } t \geq T \text {. }
$$

In particular, since $m=\left(t-\delta_{1}\right) / 2 \pi$ and $\left|\delta_{1}\right| \leq \pi$, we have

$$
\frac{2 m}{2 m-1} \leq \frac{t+\pi}{t-2 \pi} \leq \frac{1-\varphi_{2}}{1-\varphi_{1}}
$$

Let also $T$ be large enough to make the following property valid:

$$
1-\cos (x) \leq \frac{c|x|}{T} \wedge|x| \leq \pi \Rightarrow \frac{\left(1-\varphi_{2}\right) x^{2}}{2} \leq 1-\cos (x)
$$

Now we have the following chain of inequalities:

$$
\begin{aligned}
& 1-\cos \left(\delta_{2}\right) \\
\leq & \left\{f(t)=0, \text { noting } e^{t}(1-\cos (t)) \geq 0\right\} \\
& \frac{c\left|\sin \left(\delta_{2}\right)\right|}{t} \\
\leq & \{\text { by }|\sin (x)| \leq|x|\} \\
& \frac{c\left|\delta_{2}\right|}{t} .
\end{aligned}
$$

Then by (6), we have

$$
1-\cos \left(\delta_{2}\right) \geq \frac{\left(1-\varphi_{2}\right) \delta_{2}^{2}}{2}
$$

Thus, combining the upper and lower bounds on $1-\cos \left(\delta_{2}\right)$ and using (5) on the last step, we have

$$
\left|\delta_{2}\right| \leq \frac{2 c}{t\left(1-\varphi_{2}\right)} \leq \frac{2 c}{(2 m-1) \pi\left(1-\varphi_{2}\right)} \leq \frac{c}{m \pi\left(1-\varphi_{1}\right)} .
$$

Second, let $\alpha \stackrel{\text { def }}{=}(1-\varepsilon)^{-1}-\left(1-\varphi_{1}\right)^{-1}>0$. Let the threshold $T$ be large enough so that

$$
e^{-t} \leq \frac{c \alpha^{2}}{4 \pi^{2} a^{2}}\left(\frac{2 \pi}{t+\pi}\right)^{2} \text { for } t \geq T
$$

and

$$
\text { if } 1-\cos (x) \leq c / e^{T} \text { and }|x| \leq \pi \text {, then } x^{2} / 4 \leq 1-\cos (x) \text {. }
$$

The following chain of inequalities holds:

$$
\begin{aligned}
& 1-\cos \left(\delta_{1}\right) \\
= & \{\text { by } f(t)=0\} \\
& \frac{c\left|\sin \left(\delta_{2}\right)\right|-t\left(1-\cos \left(\delta_{2}\right)\right)}{e^{t}} \\
\leq & \left\{\text { by }\left|\sin \left(\delta_{2}\right)\right|,\left|\cos \left(\delta_{2}\right)\right| \leq 1\right\} \\
& \frac{c}{e^{t}} \\
\leq & \{\text { by }(7)\} \\
& \frac{c^{2} \alpha^{2}}{4 \pi^{2} a^{2}}\left(\frac{2 \pi}{t+\pi}\right)^{2} \\
\leq & \left\{\text { by }\left|\delta_{1}\right| \leq \pi\right\} \\
& \frac{c^{2} \alpha^{2}}{4 \pi^{2} a^{2}}\left(\frac{2 \pi}{t-\delta_{1}}\right)^{2} \\
= & \left\{t=2 \pi m+\delta_{1}\right\} \\
& \frac{c^{2} \alpha^{2}}{4 \pi^{2} a^{2} m^{2}} .
\end{aligned}
$$

Moreover, as $1-\cos \left(\delta_{1}\right) \leq c e^{-t} \leq c e^{-T}$, by [8), we have

$$
1-\cos \left(\delta_{1}\right) \geq \frac{\delta_{1}^{2}}{4}
$$

so combining the lower and upper bound on $1-\cos \left(\delta_{1}\right)$, we can conclude

$$
\left|a \delta_{1}\right| \leq \frac{c \alpha}{\pi m}
$$

Finally, by the triangle inequality and the bounds on $\left|a \delta_{1}\right|$ and $\left|\delta_{2}\right|$, we have

$$
\begin{aligned}
\left|a-\frac{n}{m}\right| & =\frac{\left|\delta_{2}-a \delta_{1}\right|}{2 \pi m} \leq \frac{\left|\delta_{2}\right|+\left|a \delta_{1}\right|}{2 \pi m} \\
& \leq \frac{c}{2 \pi^{2} m^{2}}\left(\alpha+\frac{1}{1-\varphi_{1}}\right)=\frac{c}{2 \pi^{2} m^{2}(1-\varepsilon)} .
\end{aligned}
$$

Now, by the premise of the Lemma, there are infinitely many $t \geq T$ such that $f(t)=0$, each yielding a pair $(n, m) \in \mathbb{N}^{2}$ which satisfies the above inequality. These infinitely many pairs $(n, m)$ witness $L_{\infty}(a) \leq c / 2 \pi^{2}(1-\varepsilon)$, as required.

Lemma 18. Fix $a \in \mathbb{R} \cap \mathbb{A}$ and $\varepsilon, c \in \mathbb{Q}$ with $a, c>0$ and $\varepsilon \in(0,1)$. If $L_{\infty}(a) \leq c(1-\varepsilon) / 2 \pi^{2}$, then $f(t)=0$ for infinitely many $t$.

Proof. We will show that there exists an effective threshold $M$, dependent on $a, c, \varepsilon$, such that if

$$
\left|a-\frac{n}{m}\right| \leq \frac{c(1-\varepsilon)}{2 \pi^{2} m^{2}}
$$

for natural numbers $n, m$ with $m \geq M$, then $f(2 \pi m) \leq 0$. Note that this is sufficient to prove the Lemma: the premise guarantees infinitely many solutions $(n, m) \in \mathbb{N}^{2}$ of 9 , so there must be infinitely many solutions with $m \geq M$, each yielding $f(2 \pi m) \leq$ 0 . Since $f$ is continuous and moreover is positive for arbitrarily large times, it must have infinitely many zeros on $[2 \pi M, \infty)$.

Now let $M$ be large enough, so that $c(1-\varepsilon) / \pi M<\pi$ and

$$
\text { if }|x|<c(1-\varepsilon) / \pi M \text {, then }(1-\varepsilon)|x| \leq|\sin (x)| \text {. }
$$

Suppose that 9 holds for $n, m \in \mathbb{N}$ with $m \geq M$ and write $t \stackrel{\text { def }}{=} 2 \pi m$. We will show that $f(t) \leq 0$. By $(9$, we have $\mid a m-$ 
$n \mid \leq c(1-\varepsilon) / 2 \pi^{2} m$. Therefore, at $=2 \pi a m=2 \pi n+\delta$ where

$|\delta| \leq c(1-\varepsilon) / \pi m<\pi$. We have

$f(t)$

$=\{$ as $\cos (t)=1\}$

$t(1-\cos (\delta))-c|\sin (\delta)|$

$\leq\left\{\right.$ by $[10)$ and $\left.1-\cos (x) \leq x^{2} / 2\right\}$

$\pi m \delta^{2}-c(1-\varepsilon)|\delta|$

$\leq\{$ by $|\delta| \leq c(1-\varepsilon) / \pi m\}$

0 . 